\title{
Validez factorial con estimación robusta de la Escala de Autoeficacia Percibida Específica de Situaciones Académicas (Eapesa) en universitarios ecuatorianos
}

Factor validity with robust estimation of the Scale of Perceived Self-efficacy Specific to Academic Situations (Eapesa) in Ecuadorian university students

\section{Validade fatorial com estimativa robusta da Escala de Autoeficácia Percebida Específica para Situações Acadêmicas (Eapesa) em estudantes universitários equatorianos}

\author{
Rodrigo Moreta-Herrera ${ }^{1}$, ORCID 0000-0003-0134-5927 \\ Carmen Montes de Oca ${ }^{2}$, ORCID 0000-0002-5743-1827 \\ Luís Fernando Navarro Cuellar ${ }^{3}$, ORCID 0000-0003-2587-6589 \\ Narciza Villegas Villacrés ${ }^{4}$, ORCID 0000-0002-1907-7361 \\ ${ }^{14}$ Pontificia Universidad Católica del Ecuador Sede Ambato, Ecuador \\ ${ }^{2}$ Universidad de los Hemisferios, Ecuador \\ ${ }^{3}$ Universidad Católica Boliviana San Pablo, Bolivia
}

\begin{abstract}
Resumen: Objetivo: Confirmar la estructura unifactorial de la Escala de Autoeficacia Percibida Específica de Situaciones Académicas (Eapesa), su fiabilidad y validez en relación con otras variables en universitarios ecuatorianos. Metodología: Estudio instrumental factorial de fiabilidad y validez de convergencia de la Eapesa. Participantes: 466 universitarios del Ecuador, $35.2 \%$ hombres y $64.8 \%$ mujeres, con edades entre 17 a 39 años $(M=21.27 ; D E=2.9)$. Resultados: Se confirma el modelo unifactorial de la Eapesa con 7 ítems que presenta un adecuado ajuste: $\chi^{2}=$ $40.01 ; p<.05 ; g l=14 ; \chi^{2} / \mathrm{gl}=2.9 ; \mathrm{CFI}=.966 ; \mathrm{TLI}=.950 ; \mathrm{SRMR}=.034 ; \mathrm{RMSEA}=.063 \mathrm{IC} 95 \%$ [.050 $.076]$ por encima de las versiones de 10 y 9 ítems. Una fiabilidad alta de $\omega=.92$ [.90 - .94] y convergencia moderada de $r=.685 ; p<.01$. Conclusiones: La Eapesa de 7 ítems es la versión de mejor rendimiento psicométrico para su aplicabilidad en universitarios del Ecuador.
\end{abstract}

Palabras clave: autoeficacia; escolaridad; análisis factorial; fiabilidad

Abstract: Objective: To confirm the unifactorial structure of the Scale of Specific Perceived SelfEfficacy of Academic Situations (Eapesa, by its acronym in Spanish), its reliability and validity in relation to other variables in Ecuadorian university students. Methodology: Eapesa's convergence validity, reliability and factorial instrumental study. Participants: 466 university students from Ecuador, $35.2 \%$ men and $64.8 \%$ women, aged between 17 to 39 years $(M=21.27 ; S D=2.9)$. Results: Eapesa's unifactorial model is confirmed with 7 items that presents an adequate adjustment: $\chi^{2}=40.01 ; p<.05 ; g l=14 ; \chi^{2} / g l=2.9$; CFI= .966; TLI= .950; SRMR= .034; RMSEA= $.063 \mathrm{IC} 95 \%[.050-.076]$ above the versions of 10 and 9 items. A high reliability of $\omega=.92[.90-.94]$ and moderate convergence of $r=.685 ; p<.01$. Conclusions: The 7-item Eapesa is the version with the best psychometric performance for its applicability in university students in Ecuador.

Keywords: self-efficacy; schooling; factor analysis; reliability 
Resumo: Objetivo: Confirmar a estrutura unifatorial da Escala de Autoeficácia Específica Percebida de Situações Acadêmicas (Eapesa), sua confiabilidade e validade em relação a outras variáveis em estudantes universitários equatorianos. Metodologia: validade de convergência da Eapesa, confiabilidade e estudo fatorial instrumental. Participantes: 466 estudantes universitários do Equador, 35,2 \% homens e 64,8 \% mulheres, com idade entre 17 e 39 anos $(M=21,27 ; D P=$ 2,9). Resultados: o modelo unifatorial da Eapesa é confirmado com 7 itens que apresentam um ajuste adequado: $\chi^{2}=40,01 ; p<0,05 ; g l=14 ; \chi^{2} / g l=2,9 ; \mathrm{CFI}=0,966 ; \mathrm{TLI}=0,950 ; \mathrm{SRMR}=0,034$; RMSEA = 0,063 IC95\% [0,050 - 0,076] acima das versões de 10 e 9 itens. Uma alta confiabilidade de $\omega=0,92[0,90-0,94]$ e convergência moderada de $r=0,685 ; p<0,01$. Conclusões: O Eapesa de 7 itens é a versão com melhor desempenho psicométrico para sua aplicabilidade em estudantes universitários em Equador.

Palavras-chave: autoeficácia; escolaridade; análise fatorial; confiabilidade

Recibido: 6/05/2020

Aceptado: 8/02/2021

Cómo citar:

Moreta-Herrera, R., Montes de Oca, C., Navarro Cuellar, L. C., \& Villegas Villacrés, N. (2021). Validez factorial con estimación robusta de la Escala de Autoeficacia Percibida Específica de Situaciones Académicas (Eapesa) en universitarios ecuatorianos. Ciencias Psicológicas, 15(1),e2153.doi:https://doi.org/10.22235/cp.v15i1.2153

Correspondencia: Rodrigo Moreta-Herrera. Pontificia Universidad Católica del Ecuador Sede Ambato, Ecuador. Email: rmoreta@pucesa.edu.ec

Para la psicología es necesario conocer y desarrollar continuamente propuestas que permitan actualizarse sobre los diversos procesos que conducen y regulan un comportamiento determinado. Desde el modelo cognitivo social, la conducta se explica por la combinación de sistemas, tanto sociales externos como internos de auto-influencia, que la modulan y regulan (Bandura, 2012). En este aspecto, la autoeficacia es importante como sistema de autoinfluencia, porque genera juicios propios positivos acerca de la capacidad resolutiva personal para elaborar procesos y alcanzar objetivos establecidos (Bandura, 1992; Suárez, García \& Moreno, 2000).

Además, la autoeficacia permite estimar la destreza propia para dominar una acción, en base a la autovaloración del potencial para culminar una meta. Lo cual se explica porque la autoeficacia permite el control y regulación del estrés que se genera por una actividad y el proceso de alcance de un objetivo propuesto (Merino Tejedor \& Lucas Mangas, 2016). De esta manera, mientras las personas tienen mayor consciencia de sus capacidades, tienen más probabilidad de desarrollar objetivos explícitos, desafiantes y llevarlos hasta su culminación. Esta percepción de capacidad de logro propia en sus distintos niveles permite por retroalimentación mejorar o empeorar los juicios y creencias sobre la eficacia personal ante un objetivo propuesto (Maddux, 2016) y por ende influir en la ejecución de conductas (Mehmood, Adnan, Shahzad, \& Shabbir, 2019). De aquí que se pone en juego la percepción de dificultad de una tarea, la capacidad resolutiva personal y la respuesta psicológica propia (Pajares, 2002).

Es importante aclarar que la autoeficacia no es un recurso generalizado de la autoinfluencia sobre todas las actividades y conductas (Luszczynska, Scholz, \& Schwarzer, 2005), sino que se condiciona por asociacionismo entre el esquema mental valoración de capacidad propia, la conducta y la respuesta social. De ahí que no necesariamente se es eficaz en toda actividad. Aun así, la evidencia actual muestra la marcada relación que guarda esta condición con distintos aspectos como: motivación voluntaria, habilidades sociales resolutivas, salud mental (Lau, Fang, Cheng, \& Kwong, 2019), también en calidad de vida, bienestar y comorbilidad (Buck et al., 2015), la 
competitividad deportiva (Jaramillo, Mayorga Lascano, \& Moreta-Herrera, 2020), la educación (Sagone \& Caroli, 2014) entre otras. Por lo que su estudio y análisis es importante para la investigación psicosocial y educativa.

\section{Autoeficacia y educación}

Ya en el campo de la educación y la psicología, el estudio a la población universitaria es necesaria debido a la particular fragilidad, vulnerabilidad (Guassi \& Telzer, 2015) y a los problemas de salud mental asociados al rendimiento que presentan (Domínguez-Lara, Prada-Chapoñan, \& Moreta-Herrera, 2019; Mayorga-Lascano \& Moreta-Herrera, 2019). En este aspecto, la autoevaluación que los universitarios tienen sobre sí mismos es relevante para la comprensión de los fenómenos psicoeducativos (Panadero, Jonsson, \& Botella, 2017). Y ahí es donde la autoeficacia (académica) juega un papel importante, debido a la implicación en la vida escolar, los estudios y las actividades académicas (Høigaard, Kovač, Øverby \& Haugen, 2015; Honicke \& Broadbent, 2016; Montes De Oca \& Moreta Herrera, 2019; Sagone \& Caroli, 2014).

La Autoeficacia Académica (AEA) es una forma específica de la autoeficacia general, que comprende las creencias y los juicios de logro personal sobre objetivos académicos (Elias \& MacDonald, 2007), como los objetivos de aprendizaje, las tareas o la superación de niveles académicos (Bandura, 1992); refiere a la autovaloración de las destrezas resolutivas sobre la formación educativa. Así, el desarrollo de actividades, contenidos, conocimientos, interés por el aprendizaje o competencias educativas como la investigación son llevados a cabo por la influencia que genera la autoeficacia (Cruz, del Rosario \& Gutiérrez Arceo, 2015).

La AEA es un área de interés y análisis por parte de la psicología educativa y la educación en general (Sagone \& Caroli, 2014). Los hallazgos contemporáneos evidencian la relación que tiene la AEA con el desempeño académico (Páez, Velásquez, Gómez \& Roldán, 2017) o con la autorregulación del aprendizaje (Wang, Shannon \& Ross, 2013), el rendimiento escolar (Richardson, Abraham \& Bond, 2012), las actitudes hacia la investigación, la resiliencia académica (Cassidy, 2015), el bienestar académico (Paciello, Ghezzi, Tramontano, Barbaranelli \& Fida, 2016), las alteraciones psicológicas en el contexto escolar (Steca et al., 2014), el estrés escolar (Chiu, 2014) entre otros. Así, el estudio de la AEA es una línea de investigación que permite explicar e intervenir en la dinámica educativa. En especial ante las dificultades que puedan atravesar los estudiantes, los docentes y el sistema educativo en general.

\section{Medición de la autoeficacia académica}

Una de las principales dificultades para la investigación psicoeducativa es la medición del constructo de autoeficacia académica. Por una parte, porque se carece de instrumental diverso y especializado para su medición; y por otra, sin la suficiente evidencia de validez e idoneidad (en especial en América Latina y en Ecuador). En el caso de la autoeficacia ligada a la escolaridad, la evaluación es frecuente con pruebas generales (Chiu, 2014; Cruz et al., 2015, Moreta-Herrera, LaraSalazar, Camacho-Bonilla \& Sánchez-Guevara, 2019; Suárez et al., 2000). Lo cual incide en errores de medida y sesgos que afectan particularmente al diagnóstico debido a la falta de especificad en el contexto académico.

En el mundo hispanohablante, una de las primeras pruebas y con más estudios es la Escala de Autoeficacia Percibida Específica de Situaciones Académicas (Eapesa; Palenzuela, 1983) desarrollada en España. Originalmente la escala se estructura unifactorialmente con 10 ítems, cuyas cargas factoriales son superiores a .63 (con excepción del ítem 9); con alta fiabilidad $\alpha=.91 \mathrm{y}$ estabilidad temporal (10 semanas) $r=.91$.

Varios estudios muestran la pertinencia de la Eapesa junto con adecuadas propiedades psicométricas que validan su uso en adolescentes y universitarios, y confirman su estructura factorial unidimensional. Así, destaca la validación en España con bachilleres y universitarios, cuya validez factorial es de $\chi^{2}=141.6 ; g l=35 ; p<.001 ; \mathrm{GFI}=.96$ y RMSEA $=.051$. Además de ser fiable 
con $\alpha=.89$, estable en intervalos de tres semanas con $r=.87$; y convergente con pruebas de metas académicas, autoconcepto académico y rendimiento (García-Fernández et al., 2010).

En Chile con adolescentes escolarizados cuyos valores de ajuste fueron de $\chi^{2}=542.4 ; g l=35$; $p<.001 ; \mathrm{GFI}=.97 ; \mathrm{CFI}=.96$ y $\mathrm{RMSEA}=.06$; con consistencia interna alta de $\alpha=.88$ y que converge con pruebas de estrategias de aprendizaje (García-Fernández et al., 2016); y en universitarios con valores de ajuste de $\chi^{2} / g l=2 ; \mathrm{CFI}=.97 ; \mathrm{TLI}=.96 \mathrm{y} \mathrm{RMSEA}=.058$ y con fiabilidad elevada de $\alpha_{(\text {ordinal })}=.87$ (Del Valle, Díaz, Pérez \& Vergara, 2018).

También en el Perú, por un lado, con estudiantes universitarios, revelando un ajuste adecuado con $\chi^{2}=64.7 ; g l=27 ; p<.001 ;$ CFI $.98 ; \mathrm{GFI}=.97$ y RMSEA $=.06$ confirmando el modelo en una versión de nueve ítems (eliminando el ítem 9), así como fiable con $\alpha=.88$ IC 90\% [.86 - .90] (Domínguez-Lara, 2014). Y, por otro lado, con adolescentes, con ajuste de $\chi^{2}=30.6 ; g l=14 ; p<$ $.001 ; \mathrm{CFI}=.99 ; \mathrm{TLI}=.99$ y RMSEA $=.06[.033-.095]$ en una versión reducida de 7 ítems $(2,3$ y 9 eliminados); con fiabilidad de $\alpha=.86$ IC 90\% [.84 - .90] (Navarro-Loli \& Domínguez-Lara, 2019).

Todos estos estudios evidencian la idoneidad de la prueba en población escolar de adolescentes y universitarios de la Eapesa por medio de un modelo unifactorial (aunque con distinto número de ítems, así como también de las opciones a disponibilidad dentro de la escala Likert de respuesta entre los ítems). En el caso de Ecuador, el modelo unifactorial no ha sido confirmado en población escolarizada, por lo que no cuenta con una validez real para su aplicabilidad. Esto tiene implicaciones psicométricas porque dificulta la realización de estudios empíricos a futuro. De este modo es necesario el desarrollo de este tipo de investigación instrumental.

\section{Consideraciones metodológicas del Eapesa}

En el estudio del Eapesa existen consideraciones metodológicas tomadas en cuenta para el desarrollo instrumental de la misma. En primer lugar, los estudios referenciales de validación reportan una baja carga factorial del ítem 9 con respecto a los ítems restantes. Esto plantea una interrogante sobre la pertinencia del ítem en el modelo factorial de la prueba y si procede o no el probar nuevas versiones reducidas con la eliminación del ítem 9 (Domínguez-Lara, 2014) o en versión reducida de 7 ítems (Navarro-Loli \& Domínguez-Lara, 2019).

También debe considerarse la metodología de confirmación de la estructura factorial en la que se presentan diferencias entre estudios. Por una parte, García-Fernández et al. (2010) y Domínguez-Lara (2014) por ejemplo, no reportan el método de extracción de factores utilizados, lo que en principio dificulta la reproducibilidad del diseño en otros ensayos. Por otra parte, GarcíaFernández et al. (2010) y Del Valle et al. (2018) reportan la estimación de Máxima Verosimilitud (MV) como metodología de factorización. Sin embargo, este método no es el más apropiado debido a la naturaleza ordinal y sin distribución normal multivariada de los ítems (Li, 2016). Finalmente, el estudio de Navarro-Loli y Domínguez-Lara (2019) utiliza el método de Mínimos Cuadrados Ponderados con Media y Varianza Ajustada (WSLMV, por su sigola en inglés), el cual es la estimación más apropiada frente a las limitaciones de la MV (Jin \& Cao, 2018), porque la escala de respuesta Likert es de cuatro opciones aun cuando la versión original es de 10 y en una versión reducida de 7 ítems (es por ello necesario el uso de la estimación de Máxima Verosimilitud Robusta). Sin embargo, para las diferencias en el uso de la metodología de factorización del Eapesa, el número de ítems empleados y el número de opciones de respuesta requieren ser aclaradas para garantizar la idoneidad de la prueba, así como la confirmación del modelo en muestra universitaria ecuatoriana, de la que no se conoce datos preliminares para el uso posterior. 


\section{Objetivos e hipótesis}

Se consideran como objetivos del estudio: a) Verificar las evidencias de validez de la estructura unifactorial del Eapesa en una muestra de universitarios ecuatorianos. Se hipotetiza que la muestra se ajusta al modelo unifactorial propuesto; b) Confirmar la fiabilidad de la escala, pues se considera que esta es alta; c) Estimar la validez del Eapesa en función de la relación con otras variables (Autoeficacia Académica). Pues se estima que el Eapesa se correlaciona con otra medida de Autoeficacia Académica.

\section{Método}

\section{Diseño}

El presente trabajo es un estudio de carácter instrumental (Ato, López, \& Benavente, 2013) en el que se busca confirmar el modelo unifactorial del Eapesa, su fiabilidad (consistencia interna) y su validez de convergencia en una muestra de universitarios del Ecuador.

\section{Participantes}

La muestra se conformó con 466 participantes (35.2\% hombres y $64.8 \%$ mujeres), con edades entre los 17 y los 39 años $(M=21.27 ; D E=2.9)$. Estos se autodefinen étnicamente como mestizos en el $93.6 \%$ y el restante $6.4 \%$ como blancos, negros e indígenas. Un $78.8 \%$ se encuentran domiciliados en los anillos urbanos de sus ciudades; mientras que el $11.2 \%$ restante en las áreas rurales.

Los participantes son estudiantes de cinco carreras (odontología, medicina, turismo, gastronomía, y contabilidad) de una universidad privada de la ciudad de Ambato, Ecuador. Un 9.4 $\%$ reciben becas y ayudas económicas y un $5.2 \%$ se encuentran en situación de riesgo académico por bajo rendimiento.

La selección de los participantes se realizó a través de un muestreo no probabilístico por conveniencia con criterios de inclusión. Estos criterios consistieron en: a) participación voluntaria; b) consentimiento firmado; c) estar en condición legal de matrícula; y d) asistencia regular a clases.

\section{Instrumentos}

Escala de Autoeficacia Percibida Específica de Situaciones Académicas (Eapesa, Palenzuela, 1983) en la versión al castellano de García-Fernández et al. (2010). Esta escala se diseñó para conocer los niveles de autoeficacia académica de los estudiantes a través de un cuestionario de 10 ítems en una escala Likert de 10 niveles desde muy desacuerdo a muy de acuerdo. Aunque no existen valores normativos específicos para la escala, se considera que a mayor puntaje existe una mayor autoeficacia percibida. El punto de corte referencial es de 50 puntos. Respecto a la fiabilidad, la consistencia interna en la versión castellana es alta con $\alpha=.89$ y temporalmente estable con $r=.87$.

Escala de Autoeficacia de Conductas Académicas (EACA; Vega, Marín, Enríquez \& Cuadras, 2011). Constituida por 13 ítems, la escala busca determinar la autoeficacia de las conductas académicas por medio de tres factores (Comunicación, Atención y Excelencia) en una escala Likert de cinco opciones de 1 (muy en desacuerdo) a 5 (muy de acuerdo). Las propiedades psicométricas de la escala presentan una fiabilidad alta en estudiantes mexicanos $(\alpha=.82$ en comunicación; $\alpha=.82$ en atención y $\alpha=.81$ en excelencia); mientras que en el presente estudio estos fueron de $\alpha=.86 ; \alpha=.91$ y $\alpha=.90$ para comunicación, atención y excelencia respectivamente.

\section{Procedimiento}

Posterior a la autorización por parte del centro educativo, se procedió a difundir el proyecto de investigación entre los estudiantes y a invitar a la participación de estos. Los alumnos leyeron y firmaron la carta de consentimiento para posteriormente realizar la evaluación en sus respectivos 
salones de clase de manera grupal bajo la supervisión de los investigadores. Este proceso duró aproximadamente 15 minutos. Luego, las evaluaciones se depuraron (se eliminaron aquellas baterías que no se encontraron plenamente llenas) y se digitalizaron en bases de datos para el tratamiento estadístico respectivo. Se verificaron las hipótesis y se construyeron reportes de investigación con los resultados alcanzados. Finalmente se elaboró el presente artículo para su difusión, que contó con la revisión de la Comisión de publicaciones de la Pucesa para garantizar la adecuación a las normas éticas en investigación.

\section{Análisis de datos}

Los análisis estadísticos se plantean en tres bloques. El primero es un examen preliminar de los ítems para conocer el comportamiento de las respuestas por medio de la media aritmética, la desviación estándar, la asimetría y la curtosis. También para verificar los supuestos de normalidad univariante, que se cumple cuando la asimetría y la curtosis se encuentran dentro del rango \pm 1.5 (Ferrando \& Anguiano-Carrasco, 2010); y también la normalidad multivariante, que existe cuando la prueba de Mardia no es significativa ( $p>$.05) (Mardia, 1970). La comprobación de estos supuestos es fundamental para el uso a futuro de los análisis de estructura factorial. Como las estimaciones robustas cuando no se cumple la normalidad multivariante.

El segundo bloque es el de estructura factorial por medio del AFC con estimación MLR (Jin \& Cao, 2018; Li, 2016). Aquí se examina el modelo unifactorial del Eapesa con 10 (Palenzuela, 1983), 9 (Domínguez-Lara, 2014) y 7 (Navarro-Loli \& Domínguez-Lara, 2019) ítems por medio de los índices de ajuste: a) Absolutos, que es el Chi cuadrado $\left(\chi^{2}\right)$, el Chi cuadrado normado $\left(\chi^{2} / g l\right)$ y el Residuo Estandarizado Cuadrático Medio (SRMR); b) Relativos, con el Índice de ajuste comparado (CFI) y el Índice de Tucker-Lewis (TLI); y c) El no basado en la centralidad que es el Error Cuadrático Medio de Aproximación (RMSEA). Se estima que el modelo factorial tiene un buen ajuste cuando el $\chi^{2}$ no es significativo ( $p>.05$ ), el $\chi^{2} / g l$ es menor a 4, el CFI y el TLI son mayores a 0.9 y el SRMR y el RMSEA son menores a 0.08 (Abad, Olea, Ponsoda, \& García, 2011; Brown, 2015; Byrne, 2006). Posteriormente se analizan las saturaciones de los ítems para conocer también el grado de aporte de los ítems al modelo. Se espera que las cargas sean superiores a $\lambda>0.5$ ya que evidencian que los ítems son consistentes y permiten una adecuada explicación de la varianza (Hair, Anderson, Tatham, \& Black, 2004).

El tercer bloque es el análisis de validez de convergencia para determinar la relación del Eapesa con el constructo de autoeficacia académica. Para ello se lo correlaciona con la EACA para conocer el grado de relación de estas. Finalmente, se verifica la fiabilidad de la prueba por medio del coeficiente omega ( $\omega$, McDonald, 1999; Trizano-Hermosilla \& Alvarado, 2016; Ventura-León \& Caycho-Rodríguez, 2017) y junto con sus intervalos de confianza al $95 \%$.

La gestión estadística de los resultados se realizó por medio del Lenguaje $\mathrm{R}$ en la versión 3.6.1. (R Core Team, 2019), asistido por los paquetes MVN, Lavaan y Mbess.

\section{Resultados}

\section{Análisis preliminar}

El análisis inicial muestra en la Tabla 1 que las medias de las respuestas a los ítems son homogéneas entre sí, con la excepción del ítem $9(M=6.38 ; D E=2.67)$ que se distancia del resto de ítems. La tendencia de respuesta se manifiesta de manera general sobre el extremo derecho de la escala, por lo que se desprende que la percepción de condiciones de autoeficacia académica está presente en la muestra de análisis.

En la verificación de la normalidad univariada y multivariada, por medio de la asimetría y curtosis, se evidencia que no todos los ítems (salvo 2 y 9) presentan distribución normal univariada. Similar situación sucede con la normalidad multivariada que no cumple con la distribución 
esperada, debido a que en la prueba de Mardia no se presencia ausencia de diferencias significativas $(p>.05)$.

Tabla 1.

Análisis descriptivo preliminar del Eapesa

\begin{tabular}{|c|c|c|c|c|}
\hline İtems & $M$ & $\overline{D E}$ & $g_{1}$ & $g_{2}$ \\
\hline $\begin{array}{l}\text { Me considero lo suficientemente capacitado para enfrentarme con } \\
\text { éxito a cualquier tarea académica. }\end{array}$ & 8.57 & 1.22 & -1.10 & 1.91 \\
\hline $\begin{array}{l}\text { Pienso que tengo bastante capacidad para comprender bien y con } \\
\text { rapidez una materia. }\end{array}$ & 8.36 & 1.27 & -0.95 & 1.44 \\
\hline $\begin{array}{l}\text { Me siento con confianza para abordar situaciones que ponen a prueba } \\
\text { mi capacidad académica. }\end{array}$ & 8.31 & 1.21 & -1.68 & 6.65 \\
\hline Tengo la convicción de que puedo hacer exámenes excelentes. & 8.52 & 1.18 & -0.92 & 1.59 \\
\hline $\begin{array}{l}\text { Me da de lado el que los profesores sean exigentes y duros, pues } \\
\text { confío mucho en mi propia capacidad académica. }\end{array}$ & 8.17 & 1.56 & -1.60 & 3.74 \\
\hline $\begin{array}{l}\text { Creo que soy una persona bastante capacitada y competente en mi } \\
\text { vida académica. }\end{array}$ & 8.40 & 1.24 & -1.40 & 4.08 \\
\hline $\begin{array}{l}\text { Si me lo propongo, creo que tengo la suficiente capacidad para } \\
\text { obtener un buen expediente académico. }\end{array}$ & 8.95 & 1.13 & -2.08 & 8.49 \\
\hline $\begin{array}{l}\text { Pienso que puedo pasar los cursos con bastante facilidad, e incluso, } \\
\text { sacar buenas notas. }\end{array}$ & 8.44 & 1.16 & -0.96 & 1.80 \\
\hline $\begin{array}{l}\text { Soy de esas personas que no necesito estudiar para aprobar una } \\
\text { asignatura o pasar un curso completo. }\end{array}$ & 6.38 & 2.67 & -0.62 & -0.58 \\
\hline $\begin{array}{l}\text { Creo que estoy preparado y bastante capacitado para conseguir } \\
\text { muchos éxitos académicos. }\end{array}$ & 8.50 & 1.41 & -1.35 & 3.54 \\
\hline \multicolumn{3}{|l|}{ Mardia } & $4260.6^{\text {*** }}$ & $113.1^{\text {*** }}$ \\
\hline
\end{tabular}

Nota: $* * * \mathrm{p}<.001 . \mathrm{g}_{1}:$ asimetría. $\mathrm{g}_{2}$ : curtosis.

\section{Análisis confirmatorio y modelos de ajuste del Eapesa}

La Tabla 2 muestra los AFC con estimación MLR en tres modelos unifactoriales del Eapesa (con 10, 9 y 7 ítems). De este análisis se desprende que el modelo propuesto de Navarro-Loli \& Domínguez-Lara (2019) de 7 ítems guarda ajustes aceptables en su estructura factorial con excepción del $\chi^{2}$ que contiene significancia $\left(p<.05\right.$, que es explicado por la sensibilidad del $\chi^{2}$ a tamaños grandes de la muestra), aunque son válidos estructuralmente una vez normalizado $\left(\chi^{2} / \mathrm{gl}\right)$ el mismo y es menor a 4. Además, los otros resultados de los indicadores de ajuste como son los absolutos $\left(\chi^{2} / g l\right.$ y SRMR), los relativos (CFI y TLI) y el no basado en la centralidad (RMSEA), muestran que presenta un mejor ajuste que el de los otros modelos (10 y 9 ítems respectivamente).

Tabla 2.

Análisis factorial confirmatorio del Eapesa con estimación MLR

\begin{tabular}{lccccccc}
\hline Modelos de ajuste & $\chi^{2}$ & $g l$ & $\chi^{2} / g l$ & CFI & TLI & SRMR & RMSEA \\
\hline 1 factor -10 ítems & $95,93^{* * *}$ & 27 & 3.6 & .951 & .935 & .037 & $.011[.089-.138]$ \\
1 factor -9 ítems & $95.93^{* * *}$ & 27 & 3.6 & .951 & .935 & .037 & $.011[.089-.138]$ \\
1 factor -7 ítems & $40.01^{* * *}$ & 14 & 2.9 & .966 & .950 & .034 & $.063[.050-.076]$ \\
\hline
\end{tabular}

Nota: ${ }^{* * *} \mathrm{p}<.001 . \chi^{2}$ : Chi Cuadrado. $\chi^{2} / \mathrm{gl}$ : Chi Cuadrado normado. CFI: Índice de Ajuste Comparado. TLI: Índice de Tucker-Lewis. SRMR: Residuo Estandarizado Cuadrático Medio. RMSEA: Error Cuadrático Medio de Aproximación. 
Junto con el AFC, se analizan las saturaciones de los ítems en la versión de 7 ítems (ítems 2, 3 y retirados; ver Figura 1). Se observa que las saturaciones son adecuadas y homogéneas entre sí, además en todos los casos son superior a $\lambda>0.5$, lo que no hace necesario el retiro de ítems; como sí en las versiones de 9 y 10 ítems y permite mayor consistencia del modelo y explicación de la varianza.

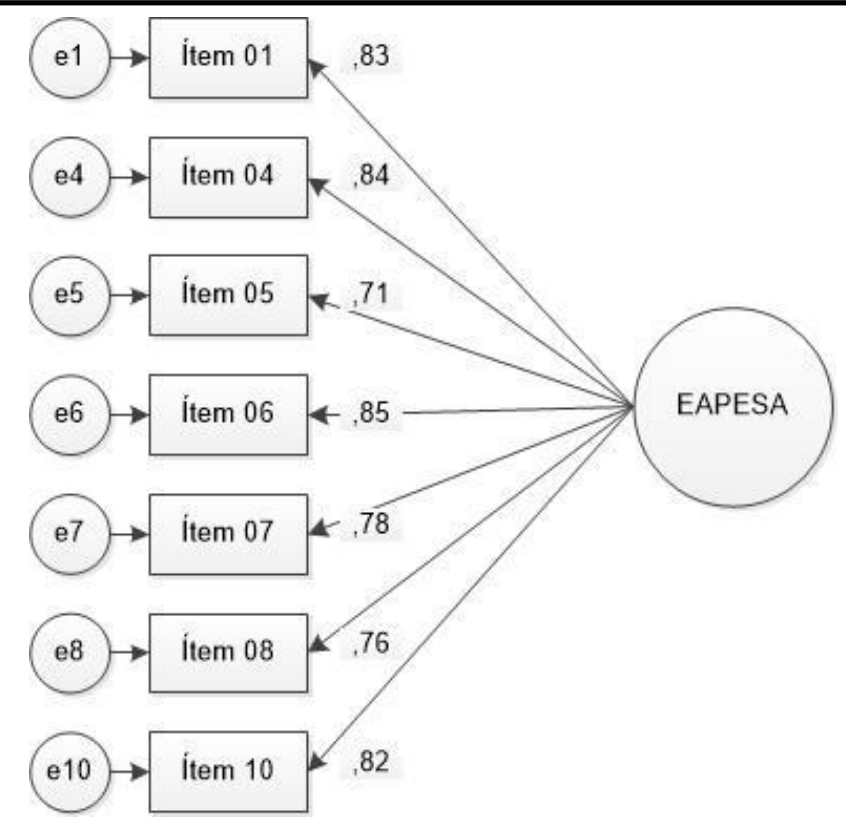

Figura 1. Estructura factorial del Eapesa con 7 ítems junto con las saturaciones de cada uno de ellos. La elipse representa al factor constitutivo, los rectángulos a los ítems y los círculos a los errores.

\section{Validez de relación con otras variables y fiabilidad}

En la Tabla 3 se analiza la relación que mantiene la Eapesa con el constructo de autoeficacia académica. Para evidenciar la validez en función de la relación con otras variables los datos de la Eapesa de 7 ítems se correlacionan con una prueba similar de autoeficacia académica, que en este caso es la EACA. Se encontró la mencionada versión del Eapesa converge de manera moderada $(r>$ $.40)$, significativa $(p<.05)$ y positiva con el EACA.

Algo similar ocurre cuando se extrae el cálculo de la fiabilidad con el coeficiente $\omega$ y los intervalos de confianza. La validez de constructo basada en la fiabilidad es alta junto con sus IC.

Tabla 3.

Análisis de validez de convergencia y fiabilidad del Eapesa en la versión de 7 ítems

\begin{tabular}{lc}
\hline Factores & Eapesa-7 \\
\hline Comunicación & $.600^{* *}$ \\
Atención & $.604^{* *}$ \\
Excelencia & $.573^{* * *}$ \\
EACA & $.685^{* *}$ \\
\hline$\omega I C_{95 \%}$ & $.92[.90-.94]$ \\
\hline Nota: ${ }^{* *} \mathrm{p}<, 01 . \omega$ IC $95 \%:$ Coeficiente de McDonald. IC $95 \%$ : Intervalos de Confianza al $95 \%$
\end{tabular}




\section{Discusión}

Los objetivos del estudio fueron confirmar el modelo unifactorial del Eapesa, así como su fiabilidad y validez de convergencia en una muestra de universitarios del Ecuador.

En primer lugar, el AFC con estimación de MLR confirma la estructura unifactorial del Eapesa propuesta por Palenzuela (1983). La versión propuesta de 7 ítems muestra un ajuste adecuado (considerando estimación robusta) en los índices absolutos $\left(\chi^{2}, \chi^{2} / g l\right.$ y SRMR), relativos (CFI y TLI) y no basado en la centralidad (RMSEA) (Abad et al., 2011; Brown, 2015; Byrne, 2006). Junto a esto, el análisis de las saturaciones de los ítems muestra cargas factoriales superiores a $\lambda>0.5$ y de manera homogénea entre sí. Esto permite estimar que los ítems contribuyen sólidamente al constructo bajo análisis y permiten una explicación de la varianza más consistente. El ajuste del Eapesa-7 en muestra ecuatoriana al ser adecuado se asemeja a los hallazgos de estructuración factorial propuesto por Navarro-Loli y Domínguez-Lara (2014) en muestra peruana y se distancia de las versiones de 10 (García-Fernández et al., 2010) y de 9 ítems (Domínguez-Lara, 2014) respectivamente.

Cabe señalar, sin embargo, que en las versiones de 7 ítems entre el análisis actual en ecuatorianos y el de Navarro-Loli y Domínguez-Lara (2019) en Perú difieren en las escalas de opción de respuesta, pues en la primera se usó 10 opciones según la versión original y 4 opciones en la segunda. Al parecer el número de opciones de respuesta entre 10 y 4 opciones no difiere en la estructuración factorial final porque se llega a la misma conclusión, sin embargo, esto debe ser tomado con cautela y considerarlo para futuras investigaciones instrumentales a fin de que se verifique el diferencial o no.

Con respecto a la fiabilidad y la validez de convergencia, se evidencia fiabilidad alta con coeficiente $\omega$. Esto concuerda con hallazgos similares en los estudios preliminares (Del Valle et al., 2018; Domínguez-Lara, 2014; García-Fernández et al., 2010; García-Fernández et al., 2016; Navarro-Loli \& Domínguez-Lara, 2019). También se presenta convergencia moderada $(r>, 40)$ y significativa $(p<.05)$ con la EACA (Vega et al., 2011). No existen datos previos que corroboren estos resultados encontrados con instrumentos similares.

A modo de conclusión, se establece que el Eapesa en la versión de 7 ítems es una versión que cuenta con estructura unifactorial adecuada, saturaciones homogéneas, fiabilidad alta y convergencia moderada válida para su aplicación en universitarios ecuatorianos. Cabe destacar que la metodología de cálculo de la factorización se llevó a cabo por medio de estimación robusta (MLR), que resulta ser más idónea para validación de pruebas con escala discreta y sin normalidad multivariada. Esto permite mayor objetividad en los resultados y menor sesgo de interpretación de la estructura factorial (Li, 2016).

Entre las implicaciones prácticas, se anota la inclusión de la población ecuatoriana en los análisis instrumentales del Eapesa que confirman la estructura factorial de esta. Además, de que se cuenta con una escala especializada de medición de la autoeficacia académica adaptada al contexto ecuatoriano, lo cual permitirá mejorar los procesos de evaluación y diagnóstico, así como de propuestas de intervención a futuro. Finalmente, permite un avance en la conformación de una prueba más exacta y precisa al proponerse una versión reducida de 7 ítems con cálculos de validación más precisos que pueden ser replicados a futuro a nivel nacional e internacional.

\section{Limitaciones y recomendaciones a futuro}

Se debe mencionar que el estudio presenta limitaciones en cuanto a la diversificación de la muestra, pues los participantes proceden de una institución educativa privada de Ecuador. Siendo a futuro necesario extender las muestras a estudiantes de universidades públicas y privadas de distintas ciudades de Ecuador con el fin de otorgarle un mayor alcance nacional. También es recomendable extender este estudio a población adolescente en bachillerato, así como al nivel de posgrado para conocer la dinámica de estructuración factorial del Eapesa. 


\section{Referencias}

Abad, F., Olea, J., Ponsoda, V., \& García, C. (2011). Medición en Ciencias Sociales y de la Salud. Madrid: Síntesis.

Ato, M., López, J., \& Benavente, A. (2013). Un sistema de clasificación de los diseños de investigación en psicología. Anales de Psicología, 29(3), 1038-1059. doi: 10.6018/analesps.29.3.178511

Bandura, A. (1992). Exercise of personal agency through the self-efficacy mechanism. En R. Schwarcer (Ed.), Self-efficacy: Thought control of action (pp. 3-38). Washington, DC: Hemisphere.

Bandura, A. (2012). Social cognitive theory. En P. Van Lange, A. W. Kruglanski, \& E. T. Higgins, Handbook of theories of social psychology (Vol. 1, pp. 349-374). London: SAGE Publications Ltd. doi: 10.4135/9781446249215.n18

Brown, T. A. (2015). Confirmatory factor analysis for applied research (Vol. 2). Nueva York: Guilford Publications.

Buck, H. G., Dickson, V. V., Fida, R., Riegel, B., D'Agostino, F., Alvaro, R., \& Vellone, E. (2015). Predictors of hospitalization and quality of life in heart failure: A model of comorbidity, self-efficacy and self-care. International Journal of Nursing, 51, 1714-1722. doi: 10.1016/j.ijnurstu.2015.06.018

Byrne, B. M. (2006). Structural equation modeling with EQS. Basic concepts, applications, and programming (ed. 2). Hills Dale, NJ: Lawrence Erlbaum Associates.

Cassidy, S. (2015). Resilience building in students: the role of academic self-efficacy. Frontiers in psychology, 6, 1781. doi: 10.3389/fpsyg.2015.01781

Chiu, S. I. (2014). The relationship between life stress and smartphone addiction on Taiwanese university student: A mediation model of learning self-efficacy and social self-efficacy. Computers in Human Behavior, 49-57. doi: 10.1016/j.chb.2014.01.024

Cruz, R., del Rosario, M., \& Gutiérrez Arceo, J. M. (2015). Sentido de autoeficacia en investigación de estudiantes de posgrado. Sinéctica, 45, 1-15.

Del Valle, M., Díaz, A., Pérez, M. V., \& Vergara, J. (2018). Análisis Factorial Confirmatorio Escala Autoeficacia Percibida en Situaciones Académicas (EAPESA) en Universitarios Chilenos. Revista iberoamericana de diagnóstico y evaluación psicológica, 4(49), 97-106. doi: 10.21865/RIDEP49.4.08

Domínguez-Lara, S. (2014). Autoeficacia para situaciones académicas en estudiantes universitarios peruanos: un enfoque de ecuaciones estructurales. Revista de Psicología-Universidad Católica San Pablo, 4(4), 45-53.

Domínguez-Lara, S., Prada-Chapoñan, R., \& Moreta-Herrera, R. (2019). Gender differences in the influence of personality on academic procrastination in Peruvian college students. Acta Colombiana de Psicología, 22(2), 137-147. doi: 10.14718/ACP.2019.22.2.7

Elias, S. M., \& MacDonald, S. (2007). Using past performance, proxy efficacy, and academic selfefficacy to predict college performance. Journal of Applied Social Psychology, 37(11), 2518-2531. doi: 10.1111/j.1559-1816.2007.00268.x

Ferrando, P. J., \& Anguiano-Carrasco, C. (2010). El análisis factorial como técnica de investigación en psicología. Papeles del Psicólogo, 31(1), 18-33.

García-Fernández, J. M., Inglés, C. J., Torregrosa, M. S., Ruiz-Esteban, C., Díaz-Herrero, Á., Pérez-Fernández, E., \& Martínez-Monteagudo, M. C. (2010). Propiedades psicométricas de la Escala de Autoeficacia Percibida Específica de Situaciones Académicas en una muestra de estudiantes españoles de Educación Secundaria Obligatoria. European Journal of Education and Psycholgy, 3(1), 61-74. doi: 10.1989/ejep.v3i1.46

Guassi, J., \& Telzer, E. (2015). Changes in family cohesion and links to depression during the college transition. Journal of Adolescents, 43, 72-82. doi:10.1016/j.adolescence.2015.05.012 
Hair, J. F., Anderson, R. E., Tatham, R. L., \& Black, W. C. (2004). Análisis multivariante. Madrid, España: Prentice Hall.

Høigaard, R., Kovač, V. B., Øverby, N. C., \& Haugen, T. (2015). Academic self-efficacy mediates the effects of school psychological climate on academic achievement. School Psychology Quarterly, 30(1), 64-74. doi: 10.1037/spq0000056

Honicke, T., \& Broadbent, J. (2016). The influence of academic self-efficacy on academic performance: A systematic review. Educational Research Review, 17, 63-84. doi: 10.1016/j.edurev.2015.11.002

Jaramillo, A., Mayorga Lascano, M., \& Moreta-Herrera, R. (2020). Ansiedad competitiva y autoeficacia en tenistas de alto rendimiento antes y después de una competencia. Revista Científica Guillermo de Ockham, 18(1), 45-54. doi: 10.21500/22563202

Jin, S., \& Cao, C. (2018). Selecting polychoric instrumental variables in confirmatory factor analysis: An alternative specification test and effects of instrumental variables. British Journal of Mathematical and Statistical Psychology, 71(2), 387-413. doi: $10.1111 / \mathrm{bmsp} .12128$

Lau, Y., Fang, L., Cheng, L. J., \& Kwong, H. K. (2019). Volunteer motivation, social problem solving, self-efficacy, and mental health: a structural equation model approach. Educational Psychology, 39(1), 112-132. doi: 10.1080/01443410.2018.1514102

Li, C. H. (2016). Confirmatory factor analysis with ordinal data: Comparing robust maximum likelihood and diagonally weighted least squares. Behavior research methods, 48(3), 936949. doi: $10.3758 / \mathrm{s} 1342$

Luszczynska, A. S., Scholz, U. \& Schwarzer, R. (2005). The general self-efficacy scale: Multicultural validation studies. The Journal of Psychology, 139(5), 439-457. doi: 10.3200/JRLP.139.5.439-457

Maddux, J. (2016). Self-efficacy. En S. Trusz, \& P. Babel (Eds.), Interpersonal and Intrapersonal Expectancies (pp. 41-46). Routeledge.

Mardia, K. (1970). Measures of Multivariate Skewness and Kurtosis with Applications Measures of Multivariate Skewness and Kurtosis with Applications. Biometrika, 57(3), 519-530. doi: $10.2307 / 2334770$

Mayorga-Lascano, M., \& Moreta-Herrera, R. (2019). Síntomas clínicos, subclínicos y necesidades de atención psicológica en estudiantes universitarios con bajo rendimiento. Revista Educación, 43(2). doi: 10.15517/REVEDU.V43I2.32239

McDonald, R. P. (1999). Test theory: A unified treatment. Mahwah: Lawrence Erlbaum Associates.

Mehmood, A., Adnan, M., Shahzad, A., \& Shabbir, F. (2019). The Effect of Self-Efficacy on Academic Performance at Higher Level of Learning: A Case Study of Punjab University Lahore. Journal of Educational Sciences, 6(1), 33-47.

Merino Tejedor, E., \& Lucas Mangas, S. (2016). La autoeficacia y la autorregulación como variables moderadoras del estrés laboral en docentes de educación primaria. Universitas Psychologica, 15(1), 205-218.

Montes De Oca, C., \& Moreta Herrera, R. (2019). La función predictora de la Autoeficacia en la Motivación Escolar en estudiantes de medicina del Ecuador. Unidades EPÍSTEME, 6(4), 565-578.

Moreta-Herrera, R., Lara-Salazar, M., Camacho-Bomilla, P., \& Sánchez-Guevara, S. (2019). Análisis factorial, fiabilidad y validez de la escala de autoeficacia general (EAG) en estudiantes ecuatorianos. Psychology, Society, \& Education, 11(2), 193-204. doi: 10.25115/psye.v11i2.2024

Navarro-Loli, J., \& Domínguez-Lara, S. (2019). Propiedades psicométricas de la Escala de Autoeficacia Percibida Específica de Situaciones Académicas en adolescentes peruanos. Psychology, Society \& Education, 11(1), 53-68. doi: 10.25115/psye.v10i1.1985 
Paciello, M., Ghezzi, V., Tramontano, C., Barbaranelli, C., \& Fida, R. (2016). Self-efficacy configurations and wellbeing in the academic context: A person-centred approach. Personality and Individual Differences, 99, 16-21. doi: 10.1016/j.paid.2016.04.083

Páez, V. C., Velásquez, D., Gómez, S., \& Roldán, C. (2017). Modelo explicativo del desempeño académico desde la autoeficacia y los problemas de conducta. Revista Colombiana de psicología, 26(1), 149-161. doi: 10.15446/rcp.v26n1.56221

Pajares, F. (2002). Gender and Perceived Self-Efficacy in Self-Regulated Learning. Theory Into Practice, 41(2), 116-125. doi: 10.1207/s15430421tip4102_8

Palenzuela, D. (1983). Construcción y validación de una escala de autoeficacia percibida específica de situaciones académicas. Análisis y Modificación de Conducta, 9(21), 185-219.

Panadero, E., Jonsson, A., \& Botella, J. (2017). Effects of self-assessment on self-regulated learning and self-efficacy: Four meta-analyses. Educational Research Review, 22, 74-98. doi: 10.1016/j.edurev.2017.08.004

R Core Team. (2019). $R$ : A language and environment for statistical computing. , $R$ Foundation for Statistical Computing. Vienna, Austria: R Foundation for Statistical Computing.

Richardson, M., Abraham, C., \& Bond, R. (2012). Psychological correlates of university students' academic performance: A systematic review and meta-analysis. Psychological bulletin, 138(2), 353-387. doi: 10.1037/a0026838

Sagone, E., \& Caroli, M. E. (2014). Locus of control and academic self-efficacy in university students: The effects of self-concepts. Procedia - Social and Behavioral Sciences, 114, 222228. doi: 10.1016/j.sbspro.2013.12.689

Steca, P., Abela, J. R., Monzani, D., Greco, A., Hazel, N. A., \& Hankin, B. L. (2014). Cognitive vulnerability to depressive symptoms in children: The protective role of self-efficacy beliefs in a multi-wave longitudinal study. Journal of abnormal child psychology, 42, 137-148. doi: 10.1007/s10802-013-9765-5

Suárez, P. S., García, A. M., \& Moreno, J. B. (2000). Escala de autoeficacia general: datos psicométricos de la adaptación para población española. Psicothema, 12(Supl.), 509-513.

Trizano-Hermosilla, I., \& Alvarado, J. M. (2016). Best alternatives to Cronbach's alpha reliability in realistic conditions: congeneric and asymmetrical measurements. Frontiers in psychology, 7, 769. doi: 10.3389/fpsyg.2016.00769

Vega, H. B., Marín, M. M., Enríquez, M. D., \& Cuadras, G. G. (2011). Análisis Psicométrico de la escala Autoeficacia en Conductas Académicas en universitarios de primer ingreso. Actualidades investigativas en educación, 11(3), 1-27. doi: 10.15517/AIE.V1113.10214

Ventura-León, J., \& Caycho-Rodríguez, T. (2017). El coeficiente Omega: un método alternativo para la estimación de la confiabilidad. Revista Latinoamericana de Ciencias Sociales, Niñez y Juventud, 15(1), 625-627.

Wang, C. H., Shannon, D. M., \& Ross, M. E. (2013). Students' characteristics, self-regulated learning, technology self-efficacy, and course outcomes in online learning. Distance Education, 34(3), 302-323. doi: 10.1080/01587919.2013.835779

Contribución de los autores: a) Concepción y diseño del trabajo; b) Adquisición de datos; c) Análisis e interpretación de datos; d) Redacción del manuscrito; e) revisión crítica del manuscrito.

R. M. H. ha contribuido en a, c, d, e; C. M. de O. ha contribuido en a, b, c, d; L. F. N. C. ha contribuido en c, d, e; y N. V. V. ha contribuido con b, d, e.

Editora científica responsable: Dra. Cecilia Cracco. 